\title{
Quantum delocalization in photon-pair generation
}

\author{
Kayn A. Forbes, Jack S. Ford, Garth A. Jones, and David L. Andrews* \\ School of Chemistry, University of East Anglia, Norwich NR4 7TJ, United Kingdom
}

(Received 26 May 2017; published 23 August 2017)

\begin{abstract}
The generation of correlated photon pairs is a key to the production of entangled quantum states, which have a variety of applications within the area of quantum information. In spontaneous parametric down-conversion-the primary method of generating correlated photon pairs - the associated photon annihilation and creation events are generally thought of as being colocated: The correlated pair of photons is localized with regards to the pump photon and its positional origin. A detailed quantum electrodynamical analysis highlights a mechanism exhibiting the possibility of a delocalized origin for paired output photons: The spatial extent of the region from which the pair is generated can be much larger than previously thought. The theory of both localized and nonlocalized degenerate down-conversion is presented, followed by a quantitative analysis using discrete-volume computational methods. The results may have significant implications for quantum information and imaging applications, and the design of nonlinear optical metamaterials.
\end{abstract}

DOI: 10.1103/PhysRevA.96.023850

\section{INTRODUCTION}

There are various methods to generate correlated photon pairs [1-3]. The most widely utilized approach is spontaneous parametric down-conversion (SPDC) [4], in which light passed through a suitable optically nonlinear medium, generates a double-wavelength output. Fundamentally a third-order electric-dipole response, the process occurs through material interactions which involve the second-order nonlinear optical susceptibility, entailing conversion of a single pump photon into a pair of phase-matched cogenerated photons. Each pair of photons generated through this mechanism has a combined energy and momentum equal to that of the corresponding annihilated photon. Significantly, they can also exhibit correlated polarizations; the same method of pair production can even lead to "hyperentangled" photon pairs $[5,6]$. When the output photon pair equally share the energy of the input, the process is known as degenerate down-conversion (DDC): Thus DDC can be alternatively viewed as the exact time reversal of second-harmonic generation [7,8]. Much interest centers on the entanglement exhibited by DDC emission.

Quantum entanglement [4,9,10] is widely regarded as the most nonclassical feature of quantum physics. The phenomenon occurs when two or more particles have mutually interdependent quantum states, such that it is only meaningful to formulate a state for the total system. Entanglement has become one of the primary means for achieving quantum operations that are forbidden with classical methods [11], commonly involving the generation of correlated photon pairs. A plethora of contemporary applications utilizing entanglement have generated the rapidly growing area of quantum information $[12,13]$. Particular disciplines that come under this umbrella term include quantum cryptography $[14,15]$, quantum teleportation [16-18], and quantum computing [19-21]. Entanglement has been demonstrated with various microscopic entities whose information obeys the quantum regime, such as neutrinos [22,23] and electrons [24]. Another active area of contemporary research involves looking at

\footnotetext{
*david.andrews@physics.org
}

entanglement between macroscopic bodies [25]. However, most theoretical and experimental work is centered upon the production and manipulation of entangled states involving two or more correlated photons [10]—with one recent experiment achieving entanglement with as many as ten photons [26]. It was with the use of correlated photon pairs that Aspect et al. performed the first experiment that violated Bell's inequalities [27-29]. Further work is centered upon their manipulation [30-32] and application [33-35], as well as the continuation of the study of entanglement at a fundamental level [36,37].

The work to be presented is concerned with further development of the quantum theory of the optically nonlinear process of SPDC [38-40], highlighting and quantifying an important contribution to the overall mechanism-the possibility of which arises from taking into account nonlocalized couplings [41]. Of course, an exact location for the creation of any output photon cannot be inferred by direct observation-although pump photon annihilation and down-converted photon emission are generally assumed to be colocated. However, the spatial extent of the region from within which any pair of downconverted photons may emerge is considerably larger than may usually be supposed. It is shown that there is a finite amplitude for each pair of correlated photons to be created at two separate locations, as indicated in Fig. 1: The mechanism proves to be mediated by a fifth-order nonlocal electric-dipole response. Accounting for such delocalized interactions provides insights concerning nonlocalization in the origin of correlated photon pairs. Moreover, allowing for each photon of the correlated pair to emerge from spatially distinct (and separated) points in space introduces a positional uncertainty of a fundamentally quantum origin. In the following analysis, utilizing a quantum electrodynamical (QED) approach cast in terms of virtual photon coupling [42], we fully account for both the localized and nonlocalized generation of correlated photon pairs. The results, cast in the form of quantum amplitudes, are further developed for computational implementation, which we then use to quantify the net effect of SPDC nonlocalization on the total rate of pair production, within a model lattice structure.

The structure of the paper is as follows. In Sec. II, the foundations of the quantum electrodynamical theory used to study SPDC in dielectric media is first laid out, beginning with 


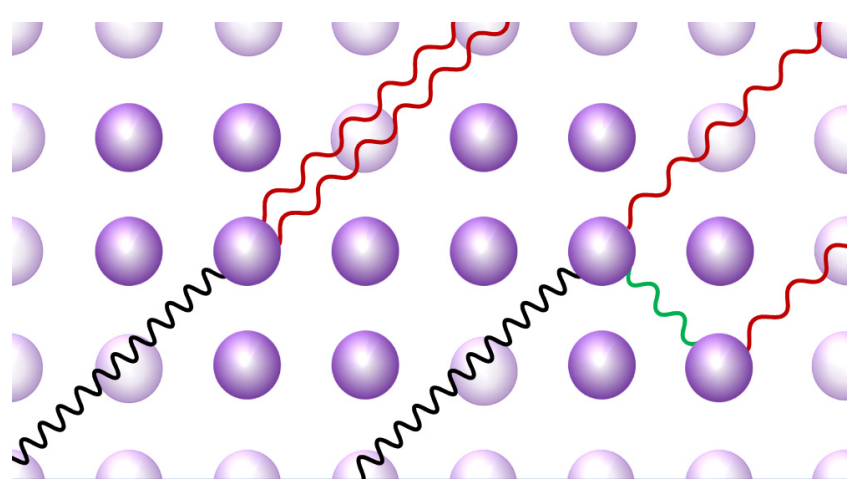

FIG. 1. The generation of correlated photon pairs (red): The lefthand side shows the localized mechanism of SPDC; the right-hand side depicts the nonlocalized mechanism.

the complete Hamiltonian used to quantify the interactions of nonrelativistic matter and the electromagnetic field. It is also shown how to develop an appropriate multicenter form of time-dependent perturbation theory, to tackle the lattice sums that arise in the later modeling. Section III deals with calculating the matrix element of localized degenerate down-conversion; Sec. IV fully develops the theory for the mechanism of nonlocalized generation of correlated photon pairs in SPDC, along with the calculation of its matrix element. Section V brings together both the matrix elements previously calculated for the localized and nonlocalized mechanisms of SPDC, in order to secure an expression for the overall rate, and in Sec. VI the result is quantitatively analyzed using a numerical lattice model. Finally, Sec. VII concludes the paper with a discussion involving the implications and applications of the full analysis.

\section{THEORETICAL FOUNDATION}

The essence of the following analysis is a treatment of mutual interaction between particles and fields in a single electrodynamical system, whereby energy is exchanged between matter and radiation through photon annihilation and creation events. In particular, the formalism of noncovariant quantum electrodynamics, commonly known as molecular QED [43-45], is adopted, as the system to be studied consists of matter possessing nonrelativistic energies. This contrasts with techniques commonly employed in studying down-conversion [46], where systems are studied using effective Hamiltonians which cast the material response in classical terms. A key advantage of QED methods is that it gives a microscopic description of the matter, including the explicit form of electrodynamic coupling between atoms or molecules. Nonrelativistic implementations of QED have been successfully employed in a wide range of optical processes and phenomena. Arguably the most well-known application of the theory is the formulation of the Casimir-Polder potential [47]—which takes due account of retardation effects. Contemporary examples of the spheres of application include optical trapping [48-52] optical binding [53-58], and optical vortices [59-62], to name but a few.

Any process of SPDC necessarily entails as a minimum three photons - to be more precise, three distinct but concerted, i.e., not separately identifiable, photon-matter interactions in the Power-Zienau-Woolley (PZW) formulation $[63,64]$ of QED. Observations of the process may in general be considered to occur within a region of material comprising $N$ optical centers, such as unit cells in a crystalline material. Each is electrically neutral and has its own electronic integrity, and the system as a whole comprises not only these centers but also the radiation field. Commonly, neglecting self-interaction terms, the system Hamiltonian is written as

$$
H=H_{\mathrm{rad}}+\sum_{\xi}^{N} H_{\mathrm{mol}}(\xi)+\sum_{\xi}^{N} H_{\mathrm{int}}(\xi) .
$$

The PZW Hamiltonian, Eq. (1), is written as a sum of three terms: (i) the second-quantized Hamiltonian for the radiation field in vacuo, $H_{\text {rad }}$; (ii) a sum over all particles with corresponding nonrelativistic Hamiltonians, $H_{\text {mol }}(\xi)$; (iii) a sum of the interaction Hamiltonians that describe the coupling between each particle and the electromagnetic field, $H_{\text {int }}(\xi)$. The subscript "mol" in the second term designates a common application to molecular systems; however, the general formalism is equally applicable to aggregates and bulk media. In this sense "mol" may be taken to represent any one of a set $\{\xi\}$ of electrically neutral material components, representative of the whole, each with a distinct locality and electronic identity: We shall commonly refer to these as "optical centers."

While the first two terms of Eq. (1) signify an "unperturbed" Hamiltonian $H_{0}$, with eigenstates suitable as a basis for a perturbation theory development, the third term describes the interaction between the radiation field and matter, accounting for the perturbative coupling and exchange of energy between the two parts of the system. This form of Hamiltonian is conveniently cast in terms of multipolar coupling [65]. (A comparison with the deployment of minimal coupling, detailing the judicious choice of framework adopted here, can be found elsewhere [66-69]). Developing the multipolar theory in expanded form, the leading term in the expansion of $H_{\text {int }}(\xi)$, using the convention of implied summation over repeated indices, is as follows:

$$
H_{\text {int }}(\xi)=-\varepsilon_{0}^{-1} \mu_{i}(\xi) d_{i}^{\perp}\left(\boldsymbol{r}_{\xi}\right),
$$

where $\mu_{i}(\xi)$ is a component of the electric-dipole operator and $d_{i}^{\perp}\left(\boldsymbol{r}_{\xi}\right)$ is a component of the transverse displacement electric field operator at the relevant position. Higher-order couplings that occur outside the electric-dipole approximation become significant when studying chiral discriminatory effects in optical processes including forces [70] and nonlinear optics [71,72].

Although the quantum field description outlined above is cast in a form best suited to describe couplings in which the material part of the system consists of only the individual optical centers involved in the interactions of interest, it can be readily expanded to account for condensed phase materials in which the interacting (active) centers are surrounded by other atoms or molecules not directly involved with the photon annihilation and creation events. This surrounding medium will, however, have electronic properties that modify the fields experienced and produced by the active optical centers, and thus play an indirect but important role in any 


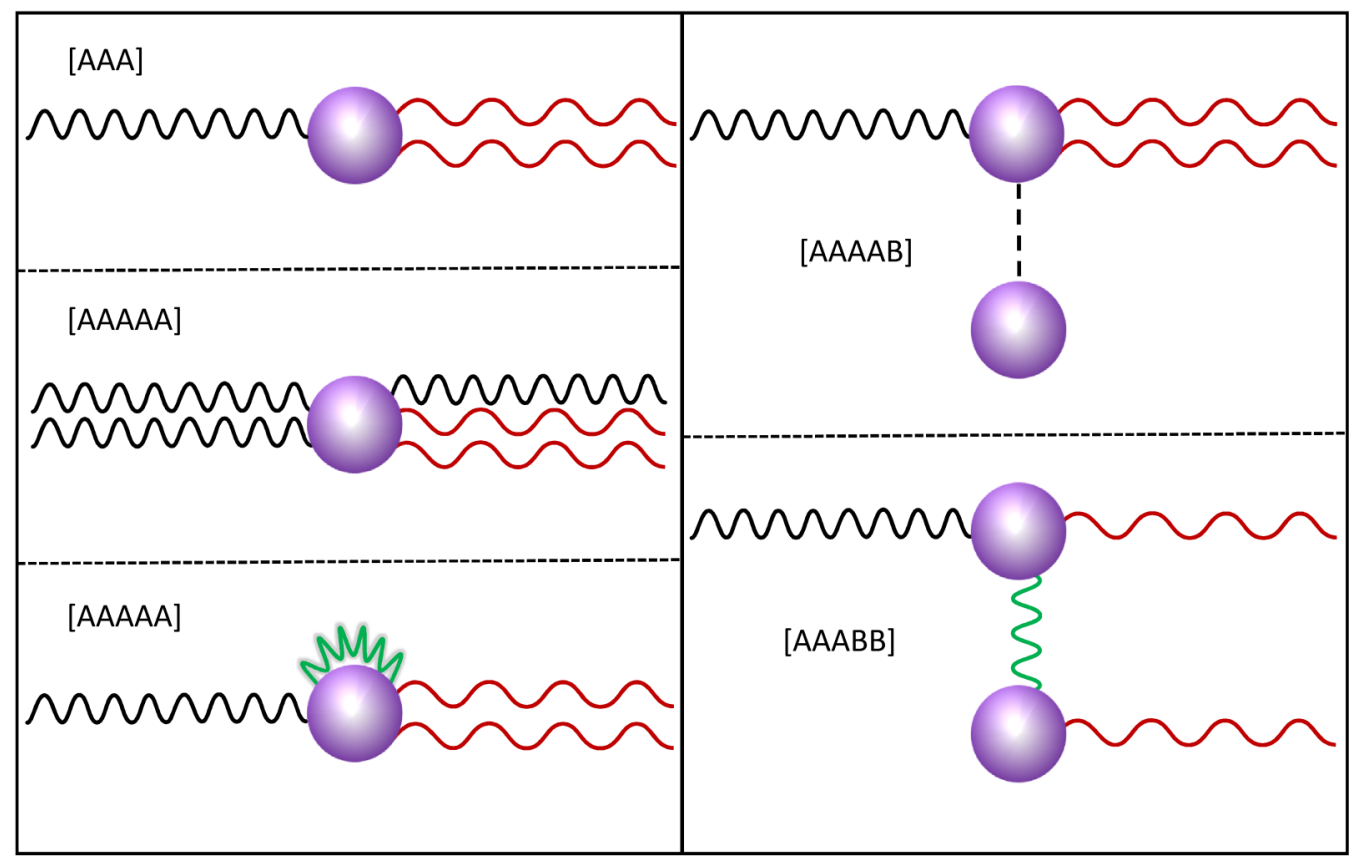

FIG. 2. Schematic representation of various optical mechanisms producing correlated photon pairs. [AAA] represents the dominant localized contribution to the rate of SPDC, while those labeled [AAAAA] are minor corrections which present no new physics; [AAAAB] highlights a static contribution to SPDC, the dotted line signifying a static electric field associated with polar components; [AAABB] depicts the nonlocalized mechanism of SPDC (virtual photon in green), the main subject matter of this paper. There also exists another mechanism [AABBB] (not shown), involving forward scattering by A prior to conventional SPDC at B; this need not concern us as it is accounted for by the inclusion of material-induced field corrections, manifest in the field expansions.

optical interaction taking place. These media corrections are accommodated within the field operators, specifically in our case the displacement electric field operator $\boldsymbol{d}^{\perp}$. This is in contrast to the semiclassical formalism whereby all Lorentz field corrections are incorporated into the optical susceptibilities [8]. Since the microscopic electric displacement field is the net field experienced by the optical centers of the medium, it can be seen as a fundamental electric field that is dynamically dressed by interaction with the surrounding electronic environment. As such, when these media effects are taken account of, the propagation and coupling of radiation in the system is properly described in terms of polaritons [73-76].

A key result for the work to be presented here is the correct form of the explicitly position-dependent electric displacement field operator $\boldsymbol{d}^{\perp}$, accounting for material-induced field corrections. A suitable form is as follows:

$$
\begin{aligned}
\boldsymbol{d}^{\perp}(\boldsymbol{r})= & i \sum_{\boldsymbol{k}, \eta}\left[\frac{\hbar \varepsilon_{0} v_{\mathrm{g}} \omega_{\boldsymbol{k}}}{2 c V n\left(\omega_{\boldsymbol{k}}\right)}\right]^{1 / 2}\left[\frac{n\left(\omega_{\boldsymbol{k}}\right)+2}{3}\right] \\
& \times\left[\boldsymbol{e}_{\boldsymbol{k}}^{(\eta)} a_{\boldsymbol{k}}^{(\eta)} e^{i \boldsymbol{k} \cdot \boldsymbol{r}}-\overline{\boldsymbol{e}}_{\boldsymbol{k}}^{(\eta)} a_{\boldsymbol{k}}^{\dagger(\eta)} e^{-i \boldsymbol{k} \cdot \boldsymbol{r}}\right],
\end{aligned}
$$

where $n\left(\omega_{k}\right)$ is the complex, frequency-dependent refractive index at polariton frequency $\omega_{k}$; the group velocity is labeled $v_{\mathrm{g}} ; V$ denotes the quantization volume; $\boldsymbol{e}$ is the polarization vector for the radiation mode $(\boldsymbol{k}, \eta)$; finally $a_{\boldsymbol{k}}^{(\eta)}$ and $a_{\boldsymbol{k}}^{\dagger(\eta)}$ designate the annihilation and creation operators, respectively, for modes of wave vector $\boldsymbol{k}$. These operate upon corresponding radiation state vectors $|q(\boldsymbol{k}, \eta)\rangle$, where $q$ is the mode occupation number (number of photons) in a mode of wave vector $\boldsymbol{k}$ and polarization $\eta$;

$$
\begin{gathered}
a_{\boldsymbol{k}}^{(\eta)}|q(\boldsymbol{k}, \eta)\rangle=\sqrt{q}|(q-1)(\boldsymbol{k}, \eta)\rangle, \\
a_{\boldsymbol{k}}^{\dagger(\eta)}|q(\boldsymbol{k}, \eta)\rangle=\sqrt{q+1}|(q+1)(\boldsymbol{k}, \eta)\rangle .
\end{gathered}
$$

The fundamentally simplest mechanism for SPDC is where all three interactions (one input photon annihilation and two output photon creations, in any sequence) occur at the same location-within the limits of uncertainty necessarily imposed by the physical extent of the unit cell. We shall label this center as A, taken to be one of $N$ identical centers within the complete system. More specifically, A is chosen as the designation of a center at which an input photon is annihilated in the course of SPDC. Accordingly, we can represent the fundamental mechanism for SPDC as [AAA], schematically depicted in Fig. 2. Now since the electric radiation field operator is linear in a sum of photon creation and annihilation operators, the lowest order that can deliver a nonvanishing result is cubic in $H_{\text {int }}$ (discounting only a very weak diamagnetic contribution). Beyond this specifically local leading term, additional contributions can only be of order $3+2 p$, where $p$ is a positive integer. Therefore, the leading corrections come from fifth order, necessarily requiring that the two additional photon events are the creation and annihilation of a photon in an arbitrary mode-i.e., a virtual photon, fleetingly borrowing energy from the background vacuum fluctuations.

The two possibilities arise: [AAAAA] and [AAABB]. The former is, as such, not separately identifiable: It signifies a self-energy correction to the fundamental process at A, with no bearing on issues of nonlocality, and may indeed be regarded as 
a minor correction (accounting for an optical Kerr effect shift in energy levels). The latter is a feature that can be identified only using a microscopic theory of light-matter interactions: It signifies a mechanism wherein SPDC is cooperatively delivered by two centers, A and B, coupled by a virtual photon that is created at one center and annihilated at the other. It delivers subtly different physics, the subject of the detailed analysis that follows. The designation [AAABB] signifies only a partition of the $N$ physical centers into $N(N-1)$ potential $\mathrm{AB}$ pairs; it does not imply any specific time ordering, since theory requires all time orderings to be taken into account. It serves to identify the most prominent source of nonlocal SPDC, associated with fifth-order perturbation theory.

We now turn to the foundational equations for the quantum amplitude $M_{f i}$, whose modulus square will determine the efficiency of the overall process [77]:

$$
\begin{gathered}
M_{f i}=\sum_{s=0}\left\langle f\left|\left(H_{\mathrm{int}} T_{0}\right)^{s} H_{\mathrm{int}}\right| i\right\rangle, \\
T_{0} \approx\left(E_{i}-H_{0}\right)^{-1},
\end{gathered}
$$

where the latter expression for the propagator $T_{0}$ is based on the eigenstates of the full Hamiltonian $H$ being similar to those of $H_{0}$. Equation (6) represents a coupling that in general propagates from an initial system state $i$ into a final state $f$, where $s$ is the order of perturbation theory based on the eigenstates of $H_{0}$. In both these equations, all states, energies, and operators relate to the system as a whole, i.e., the material as a whole plus the radiation field. For a wave-vector-matched process such as SPDC, the optical center sums that feature in the quantum amplitude (6) will be phase coherent and therefore directly additive. Noting that only even values of $s \geqslant 2$ can contribute to the sum in Eq. (6), then on implementing the optical center sums we obtain a result expressible as

$$
\begin{aligned}
M_{f i}= & \sum_{p=0}\left\langle f_{\mathrm{rad}}\right| \prod_{j=1}^{N}\left\langle 0\left(\xi_{j}\right)\right|\left[\sum_{k=1}^{N} H_{\mathrm{int}}\left(\xi_{k}\right) T_{0}\right]^{2(p+1)} \\
& \times \sum_{m=1}^{N} H_{\mathrm{int}}\left(\xi_{m}\right) \prod_{l=1}^{N}\left|0\left(\xi_{l}\right)\right\rangle\left|i_{\mathrm{rad}}\right\rangle .
\end{aligned}
$$

The leading term, $p=0$, delivers interactions that are third order in $H_{\text {int }}$, comprising a sum of $N$ terms, each signifying single-center SPDC. To this order, it is readily established that all terms for which the three operations of $H_{\text {int }}$ are not effected at the same center vanish-as, of course, energy conservation must require. With the use of the completeness relation

$$
1=\sum_{r}\left|r\left(\xi_{q}\right)\right\rangle\left\langle r\left(\xi_{q}\right)\right|
$$

for any individual center $\xi_{q}$, the leading term to emerge from Eq. (8) takes the form

$$
M_{f i}^{(3)}=\sum_{r^{(2)}, r^{(1)}} \frac{\left\langle f\left|H_{\text {int }}\right| r^{(2)}\right\rangle\left\langle r^{(2)}\left|H_{\text {int }}\right| r^{(1)}\right\rangle\left\langle r^{(1)}\left|H_{\text {int }}\right| i\right\rangle}{\left(E_{i}-E_{r^{(2)}}\right)\left(E_{i}-E_{r^{(1)}}\right)},
$$

where it is implicit that the entire expression relates to a single, representative center. Equation (10) thus relates to all $N$ conventional SPDC interactions of type [AAA] identified above. The next order, $p=1$ in Eq. (8), delivers the leading corrections, of the form [AAAAA] and [AAABB]. By similar arguments to those used above, the following emerges as a representative of the former:

$$
M_{f i}^{(5)}=\sum_{r^{(4)}, r^{(3)}, r^{(2)}, r^{(1)}} \frac{\left\langle f\left|H_{\mathrm{int}}\right| r^{(4)}\right\rangle\left\langle r^{(4)}\left|H_{\mathrm{int}}\right| r^{(3)}\right\rangle\left\langle r^{(3)}\left|H_{\mathrm{int}}\right| r^{(2)}\right\rangle\left\langle r^{(2)}\left|H_{\mathrm{int}}\right| r^{(1)}\right\rangle\left\langle r^{(1)}\left|H_{\mathrm{int}}\right| i\right\rangle}{\left(E_{i}-E_{r^{(4)}}\right)\left(E_{i}-E_{r^{(3)}}\right)\left(E_{i}-E_{\left.r^{(2)}\right)\left(E_{i}-E_{r^{(1)}}\right)}\right.} .
$$

The expression signifies a self-energy [AAAAA] correction to single-center SPDC, all interactions once again occurring at the same center. However, the $p=1$ term in Eq. (8) can also deliver nonvanishing results when two distinct centers are involved. Then, the completeness relation of Eq. (9) is to be implemented not only for any center taking the role of A, but also in each case for the full set of $(N-1)$ centers that can act as component B. Accordingly one can write

$$
1=(N-1)^{-1} \sum_{q \neq \mathrm{A}}^{N-1} \sum_{r}\left|r\left(\xi_{q}\right)\right\rangle\left\langle r\left(\xi_{q}\right)\right|
$$

This signifies that a normalization factor of $1 /(N-1)$ has to be included in implementing a global sum over all centers that can take the role of B. This factor will arise once in each state sequence, at the point where the operation of $H_{\text {int }}$ is first effected at center B. To summarize, we can thus write

$$
\begin{aligned}
M_{f i}= & \sum_{\xi}^{N} M_{f i}^{(3)}(\xi)+\sum_{\xi}^{N} M_{f i}^{(5)}(\xi) \\
& +(N-1)^{-1} \sum_{\xi}^{N} \sum_{\xi^{\prime}}^{N-1} M_{f i}^{(5)}\left(\xi, \xi^{\prime}\right)+\cdots,
\end{aligned}
$$

where the three successive terms represent explicit calculations for quantum amplitude contributions of the form [AAA], [AAAAA], and [AAABB].

To proceed further we need to be able to follow the time development of the state of the closed dynamical system at any time, given its initial conditions. Herein, we employ the Fermi rate to calculate the desired quantum rates:

$$
\Gamma_{\delta}=\frac{2 \pi}{\hbar}\left|M_{f i}\right|^{2} \delta\left(\hbar \omega-2 \hbar \omega^{\prime}\right),
$$


where the Dirac delta function ensures energy conservation: The sum of the energies for the two emergent photons, $2 \hbar \omega^{\prime}$, equals that of the input photon, $\hbar \omega$. The subscript on the lefthand side of Eq. (14) signifies that the observed rate $\Gamma$ will, in practice, be secured on integrating over all energy-conserving outputs.

To evaluate quantum amplitudes using the perturbative expansion of Eq. (13) and ultimately the measurable rates with the aid of Eq. (14), diagrammatic techniques are used to reduce the computational complexity of the calculations. Such tools also provide valuable insight into the underlying physical mechanisms by providing a visual representation of the photon-matter interactions. The most widely known and commonly deployed of these methods are the time-ordered graphs of Feynman [78-80]. As far as the radiation states are concerned, summation over all intermediate states that link the same initial and final states is equivalent to drawing all the possible topologically distinct Feynman diagrams for that particular given series of photon annihilation and/or creation events. However, the relative drawback to these time-ordered diagrams is that for higher-order processes involving many photon annihilation and creation events, or processes involving multiple optical centers, the number of possible Feynman diagrams describing all the possible pathways from initial to final states can become daunting. To overcome this, the statesequence method [81] has been developed as an alternative diagrammatic technique to time-ordered diagrams. As will be shown by its deployment in Sec. IV, a state-sequence diagram encapsulates all possible intermediate states that link the initial and final states, with each pathway signifying a single time ordering; thus, a single state-sequence diagram contains all time orderings for any given process.

\section{LOCALIZED SPDC}

In the theory of quantum electrodynamics, the generation of correlated photon pairs by SPDC occurs through the annihilation of a pump photon possessing a wave vector $\boldsymbol{k}$ and the creation of two output photons of wave vector $\boldsymbol{k}^{\prime}$, where $\boldsymbol{k}=2 \boldsymbol{k}^{\prime}$ and hence the output is coherent in character. Indeed, the pair of down-converted photons not only conserve
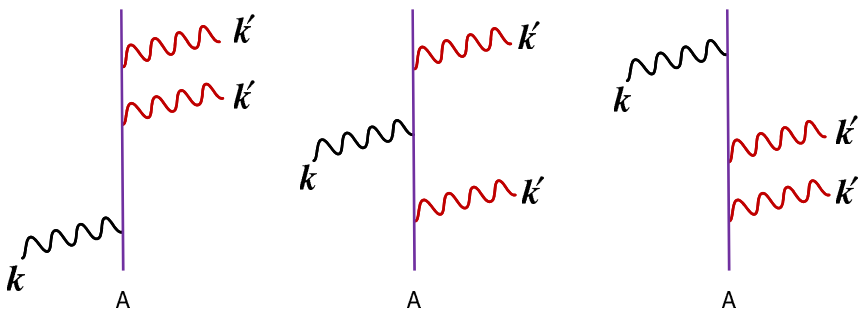

FIG. 3. The three topologically distinct Feynman time-ordered diagrams representing the degenerate down-conversion of a pump photon with wave vector $\boldsymbol{k}$ into a pair of correlated photons with wave vector $\boldsymbol{k}^{\prime}$ at a single, physically distinct center A.

the energy and momentum of the original input photon in degenerate down-conversion; they also exhibit correlated polarization states. Traditionally, the positional range of the location for the emission of down-converted photons and input photon annihilation is assumed to be small-certainly well within the span of a wavelength. It would usually be assumed that the process of SPDC occurs in an essentially localized, colocated fashion, where only the diffuse nature of atomic and molecular orbitals limits the precision with which the location for any photon creation event can be linked to a site of input photon annihilation.

Using the methods outlined in Sec. II, we now proceed to calculate the quantum amplitude for single-center, localized SPDC. As indicated in the previous section, the three photonmatter interactions that are intrinsic to the nature of SPDC lead to a formulation in time-dependent perturbation theory whose leading, third-order term is associated with three distinct time orderings, as exhibited in Fig. 3.

The initial and final states of the system are $|i\rangle=\left|E_{0}^{A} ; \hbar c k\right\rangle$ and $|f\rangle=\left|E_{0}^{A} ; 2 \hbar c k^{\prime}\right\rangle$, respectively, where both the initial and final states of the material system are ground states. Considering a single interaction at position $\mathrm{A}$ in a suitable nonlinear optical medium and, for reasons outlined above, applying the electric-dipole approximation with Eq. (10) and the first term in Eq. (2) gives the result of Eq. (15) below-in which the three terms within the square brackets correspond to the three time orderings exhibited in Fig. 3.

$$
\begin{aligned}
M_{\mathrm{A}}= & \frac{i}{\varepsilon_{0}^{3}} \sum_{\boldsymbol{k}^{\prime}, \eta^{\prime}} \sqrt{q}\left[\frac{\hbar \varepsilon_{0} v_{g} \omega_{\boldsymbol{k}}}{2 c V n\left(\omega_{\boldsymbol{k}}\right)}\right]^{1 / 2}\left[\frac{\hbar \varepsilon_{0} v_{g}^{\prime} \omega_{\boldsymbol{k}^{\prime}}^{\prime}}{2 c V n\left(\omega_{\boldsymbol{k}^{\prime}}^{\prime}\right)}\right]\left[\frac{n^{2}\left(\omega_{\boldsymbol{k}}\right)+2}{3}\right]\left[\frac{n^{2}\left(\omega_{\boldsymbol{k}^{\prime}}^{\prime}\right)+2}{3}\right]^{2} e_{i}^{(\eta)}(\boldsymbol{k}) \bar{e}_{j}^{\left(\eta^{\prime}\right)}\left(\boldsymbol{k}^{\prime}\right) \bar{e}_{k}^{\left(\eta^{\prime}\right)}\left(\boldsymbol{k}^{\prime}\right) \\
& \times \sum_{r, s}\left[\frac{\mu_{i}^{0 r} \mu_{j}^{r s} \mu_{k}^{s 0}}{\left(E_{s 0}-\hbar c k^{\prime}\right)\left(E_{r 0}-2 \hbar c k^{\prime}\right)}+\frac{\mu_{j}^{0 r} \mu_{i}^{r s} \mu_{k}^{s 0}}{\left(E_{s 0}-\hbar c k^{\prime}\right)\left(E_{r 0}+\hbar c k^{\prime}\right)}+\frac{\mu_{j}^{0 r} \mu_{k}^{r s} \mu_{i}^{s 0}}{\left(E_{s 0}+2 \hbar c k^{\prime}\right)\left(E_{r 0}+\hbar c k^{\prime}\right)}\right] e^{i\left(\boldsymbol{k}-2 \boldsymbol{k}^{\prime}\right) \cdot \boldsymbol{r}_{A}} .
\end{aligned}
$$

Assuming no down-converted photons are initially present, i.e., $q^{\prime}=0$, in principle demands retention of the sum over the output mode $\left(\boldsymbol{k}^{\prime}, \eta^{\prime}\right)$. However, the conservation of photon momentum is intrinsic to the coherent process; i.e., $\boldsymbol{k}=2 \boldsymbol{k}^{\prime}$ for SPDC. The optical frequency of the input is such that $n(\omega) \omega=c k \equiv c|\boldsymbol{k}|$, and for the output $n\left(\omega^{\prime}\right) \omega^{\prime}=c\left|\boldsymbol{k}^{\prime}\right|$. This means that for SPDC to occur at any sizable rate in the condensed phase, the process must occur with index matching-and for simplicity we can neglect any minor difference in refractive index at frequencies $\omega$ and $\omega^{\prime}$ : The index therefore appears as simply $n$ below. In the absence of significant optical dispersion, group velocity reduces to phase velocity such that $v_{g}=c / n$. Eq. (15) can be further modified to highlight the role that the frequency-dependent nonlinear-susceptibility $\chi^{(2)}(\omega)[8]$ takes in SPDC, the form of which 
is conventionally cast in tensor form as follows:

$$
\begin{aligned}
& \chi_{i(j k)}^{(2)}(-\omega,-\omega ; 2 \omega) \\
& \quad=\frac{N^{\prime}}{2 \varepsilon_{0}}\left(\frac{n^{2}+2}{3}\right)^{3} \sum_{r, s}\left[\left\{\frac{\mu_{i}^{0 r} \mu_{j}^{r s} \mu_{k}^{s 0}}{\left(E_{s 0}-\hbar \omega\right)\left(E_{r 0}-2 \hbar \omega\right)}+\frac{\mu_{j}^{0 r} \mu_{i}^{r s} \mu_{k}^{s 0}}{\left(E_{s 0}-\hbar \omega\right)\left(E_{r 0}+\hbar \omega\right)}+\frac{\mu_{j}^{0 r} \mu_{k}^{r s} \mu_{i}^{s 0}}{\left(E_{s 0}+2 \hbar \omega\right)\left(E_{r 0}+\hbar \omega\right)}\right\}+\{j \leftrightarrow k\}\right],
\end{aligned}
$$

where $N^{\prime}$ is the number density of active centers, equivalent to $V^{-1}$, and $\{j \leftrightarrow k\}$ signifies the other terms by exchanging these indices. Taking all the above into consideration, Eq. (15) can be written more succinctly as

$$
M_{\mathrm{A}}=\frac{i}{4}\left(\frac{\hbar \omega}{n^{2}}\right)^{3 / 2}\left(\frac{q}{\varepsilon_{0} V}\right)^{1 / 2} e_{i} \bar{e}_{j}^{\prime} \bar{e}_{k}^{\prime} \chi_{i(j k)}^{(2)}(-\omega,-\omega, 2 \omega)
$$

The product polarization tensor $e_{i} \bar{e}_{j}^{\prime} \bar{e}_{k}^{\prime}$ in Eq. (15) is $j, k$ symmetric, and therefore in its contraction with the nonlinearsusceptibility tensor Eq. (16), only the $j, k$-symmetric part of the latter contributes. The above equation (17), signifying the matrix element for single-center degenerate down-conversion within a dielectric medium, now represents a basis from which to develop the theory of nonlocalized down-conversion.

\section{NONLOCALIZED SPDC}

As intimated earlier, nonlocalized contributions to the overall rate of SPDC entail a secondary mechanism involving the coupling of point A with a new point in the optical medium, which we label B. No constraints are imposed on the relative positions of $\mathrm{A}$ and $\mathrm{B}$ - at a later stage in the theory, a sum is to be effected over all sites that can fulfill B's role. We assume that $\mathrm{A}$ and $\mathrm{B}$ are electronically equivalent, so that the labeling of $\mathrm{A}$ and $\mathrm{B}$ is arbitrary-the former being associated only with the position of input photon annihilation. For this reason there is no need to entertain role reversal $\mathrm{A} \leftrightarrow \mathrm{B}$. The mechanism for the delocalized form of SPDC is as follows: A pump photon of frequency $\omega$ is annihilated at point $A$ (as in the localized mechanism derived above in Sec. III); however, A only emits a single real photon of frequency $\omega^{\prime}$ while it also couples to B, via virtual photon propagation-B itself emitting another single real photon of frequency $\omega^{\prime}$. The correlated photon pair thus emerges from two, delocalized points within the medium.

The delocalized mechanism entails five photon-matter interaction events (Fig. 4), so we now have recourse to Eq. (11) for the corresponding quantum amplitude. The initial and final states are the same as in the previous section, but now position $\mathrm{B}$ is specifically included as a constituent of the system $|i\rangle=$ $\left|E_{0}^{A} ; E_{0}^{B} ; \hbar c k\right\rangle$ and $|f\rangle=\left|E_{0}^{A} ; E_{0}^{B} ; 2 \hbar c k^{\prime}\right\rangle$. The sum over all intermediate virtual states $r^{(1)} \cdots r^{(4)}$ naturally decomposes into a sum over $5 !=120$ time-ordered permutations of the five interaction events, each of which delivers a distinct form for the quantum amplitude's energy-difference denominator (see Fig. 5).

To exemplify the calculation, if we initially choose just one of these time orderings-say, the upper pathway in Fig. 5-then the sum over system states reduces to a sum over intermediate states for the sites A and B and a sum over radiation modes for the virtual photon. The result for this pathway is as follows:

$$
\begin{aligned}
M_{\mathrm{AB}(1)}= & \frac{i}{4 N^{\prime}} \sum_{r, s} \sum_{\boldsymbol{p}, \phi}\left(\frac{\hbar c k}{n^{2}}\right)^{3 / 2}\left(\frac{\hbar c p}{\varepsilon_{0} V n^{2}}\right)\left(\frac{q \varepsilon_{0}}{V}\right)^{1 / 2}\left(\frac{n^{2}+2}{3}\right)^{5} e_{i}{\overline{e^{\prime}}}_{k}{\overline{e^{\prime}}}_{m} \bar{e}_{l}^{(\phi)} e_{j}^{(\phi)} \mu_{m}^{r 0(\mathrm{~B})} \mu_{k}^{r 0(\mathrm{~A})} \mu_{l}^{s r(\mathrm{~A})} \mu_{i}^{0 s(\mathrm{~A})} \mu_{j}^{0 r(\mathrm{~B})} \\
& \times\left\{\left(E_{0 r}^{B}-\hbar c k^{\prime}\right)\left(E_{0 r}^{A}+E_{0 r}^{B}-2 \hbar c k^{\prime}\right)\left[E_{0 s}^{A}+E_{0 r}^{B}-\hbar c\left(2 k^{\prime}+p\right)\right]\left(E_{0 r}^{B}-\hbar c p\right)\right\}^{-1} e^{i\left(\boldsymbol{p}-\boldsymbol{k}^{\prime}\right) \cdot \boldsymbol{r}_{B}}
\end{aligned}
$$

We have chosen the position A to be the origin of our spatial coordinate system, so the positional vector $\boldsymbol{r}_{\mathrm{B}}$ is the displacement of site B from site A. The virtual photon has wave vector $\boldsymbol{p}$ and polarization state $\phi$, but these will not appear as variables in the final result, as all of their possible values are included in the sum [82]. Combining the result of Eq. (18) with the other 119 terms for the other state sequences in Fig. 5 yields the full nonlocalized quantum amplitude:

$$
M_{\mathrm{AB}}=\frac{i}{4 N^{\prime}}\left(\frac{\hbar \omega}{n^{2}}\right)^{3 / 2}\left(\frac{q \varepsilon_{0}}{V}\right)^{1 / 2} \frac{1}{n^{2}} \mathrm{e}^{-i \boldsymbol{k}^{\prime} \cdot \boldsymbol{r}_{\mathrm{B}}} e_{i}{\overline{e^{\prime}}}_{k}{\overline{e^{\prime}}}_{m} \chi_{i(j k)}^{(2)}(-\omega,-\omega ; 2 \omega) \chi_{l m}^{(1)}(-\omega ; \omega) V_{j l}\left(n, k^{\prime}, \boldsymbol{r}_{\mathrm{B}}\right) .
$$

As with the single-center contribution, Eq. (17), the above equation succinctly expresses the nonlinear-susceptibility and other tensors as known functions: the linear susceptibility,

$$
\chi_{l m}^{(1)}(-\omega, \omega)=\frac{N^{\prime}}{\varepsilon_{0}}\left(\frac{n^{2}+2}{3}\right)^{2} \sum_{r}\left[\frac{\mu_{l}^{0 r} \mu_{m}^{r 0}}{E_{r 0}^{\mathrm{B}}+\hbar \omega}+\frac{\mu_{m}^{0 r} \mu_{l}^{r 0}}{E_{r 0}^{\mathrm{B}}-\hbar \omega}\right]
$$


and the retarded resonance electric-dipole coupling [83],

$$
V_{j l}\left(n, k^{\prime}, \boldsymbol{r}_{\mathrm{B}}\right)=\frac{\mathrm{e}^{i n k^{\prime} r_{\mathrm{B}}}}{4 \pi \varepsilon_{0} r_{\mathrm{B}}^{3}}\left[\left(\delta_{j l}-3 \hat{r}_{\mathrm{B} j} \hat{r}_{\mathrm{B} l}\right)\left(1-i n k^{\prime} r_{\mathrm{B}}\right)-\left(\delta_{j l}-\hat{r}_{\mathrm{B} j} \hat{r}_{\mathrm{B} l}\right) n^{2} k^{\prime 2} r_{\mathrm{B}}^{2}\right] .
$$

In Eq. (21), $n$ is the complex refractive index. Fully engaging the complex value enables dissipative effects to be incorporated in the theory without contriving an additional damping factor.

\section{PAIR-GENERATION RATE}

With the results of the previous two sections we now have the matrix elements for both the localized Eq. (17) and nonlocalized Eq. (19) mechanisms, together applicable within the Fermi rule Eq. (14) in order to determine an overall down-conversion rate. Since the amplitudes $M_{\mathrm{A}}$ and $M_{\mathrm{AB}}$ are terms of Eq. (13) for a specific site $\xi$ labeled A and a second $\xi^{\prime}$ labeled B, the amplitude argument in Eq. (14) becomes a sum over all pairs of sites $\mathrm{A}$ and $\mathrm{B}$,

$$
M_{f i} \rightarrow \sum_{\mathrm{A}}^{N}\left(M_{\mathrm{A}}+\sum_{\mathrm{B} \neq \mathrm{A}}^{N-1} M_{\mathrm{AB}}\right) .
$$

The $M_{\mathrm{A}}$ term in principle has both third- and fifth-order contributions, but the latter can be ignored as it is a minor correction adding no new physics (see Fig. 2). Our focus is therefore upon the modification to $M_{f i}$ wrought by including the nonlocal contributions, $M_{\mathrm{AB}}$. Substitution of Eqs. (17) and (19) into Eq. (22) and using the Fermi rule, Eq. (14), gives a rate equation for SPDC modified by delocalization:

$\Gamma=S\left|e_{i} \bar{e}_{k}^{\prime} \chi_{i(j k)}^{(2)}\left[\bar{e}_{j}^{\prime}+\bar{e}_{m}^{\prime} \chi_{l m}^{(1)} \frac{\varepsilon_{0}}{n^{2} N^{\prime}(N-1)} \sum_{B}^{N-1} \mathrm{e}^{-i \boldsymbol{k}^{\prime} \cdot \boldsymbol{r}_{B}} V_{j l}\right]\right|^{2}$.

The tensor functions have been written without their arguments for brevity. All scalar factors common to both terms

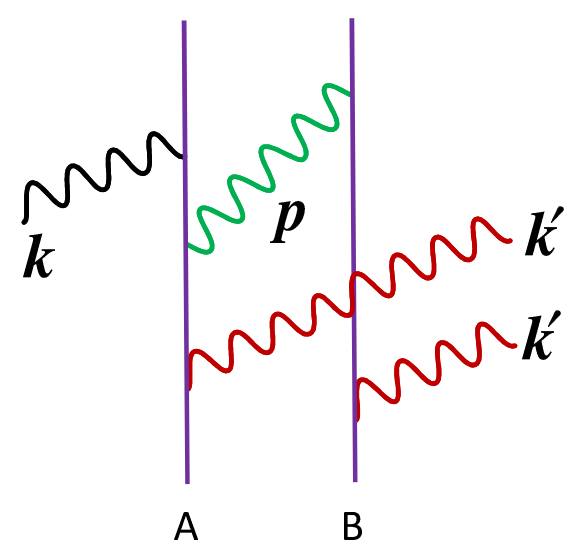

FIG. 4. One of the 120 Feynman time-ordered diagrams representing nonlocalized generation of correlated photon pairs of wave vector $\boldsymbol{k}^{\prime}$. This particular diagram represents the upper pathway in Fig. 5, and the single contribution to the total matrix element represented by Eq. (18). are consolidated into an undefined constant of proportionality $S$. Notably, the amplitude $M_{f i}$ has the same form for every site A, so the sum over all positions of A becomes a factor of $N^{2}$ hidden within $S$.

The SPDC rate $\Gamma$ is calculated by performing a global sum of all sites B, including every possible interacting site in the medium other than the chosen A. In a solid medium sample any larger than a few micrometers this is an unfeasible number of terms to include, so we only include sites that lie within a certain cutoff distance from the point A. Each active site of the medium is only included in the summation over $\mathrm{B}$ if $r_{\mathrm{B}} \leqslant C$, such that $C$ is the cutoff radius of a spherical region centered on A, within which nonlocalization is to be accounted. By calculating the rate as a function of this parameter $C$, we can determine the relative contribution of sites $\mathrm{B}$ at various distances from A.

The first step is to formulate the summation over B with $(N-1)$ terms as a function of $C$, yielding a tensor labeled $\sigma_{j l}$.

$$
\sigma_{j l}(C) \equiv \sum_{B}^{N_{C}} \mathrm{e}^{-i \boldsymbol{k}^{\prime} \cdot \boldsymbol{r}_{B}} V_{j l}
$$

The integer $(N-1)$ in Eq. (23) becomes $N_{C}$, equal to the number of centers B obeying $r_{B} \leqslant C$. Expressed as a function of the cutoff $C$, the rate equation (23) then becomes

$$
\Gamma_{C}=S\left|e_{i} \bar{e}_{k}^{\prime} \chi_{i(j k)}^{(2)}\left(\bar{e}_{j}^{\prime}+\frac{\varepsilon_{0}}{n^{2} N^{\prime}} \bar{e}_{m}^{\prime} \chi_{l m}^{(1)} \frac{\sigma_{j l}}{N_{C}}\right)\right|^{2} .
$$

The expansion of the system from a single center to a spherical region of radial cutoff $C$ has implications for interpreting the overall rate of observed pair emission. The observable rate is increased by the cross-section area of the active region. Rate equation (25) describes the downconversion from an individual center $\mathrm{A}$, but by allowing for emission of the second photon from any B within the sphere, it is implicit that we are measuring emission over this circular area. Hence the effective observable rate, $\Gamma_{C}^{\prime}$, is given by multiplying the second term by the cross-section area of the sphere, $\pi C^{2}$ - since this is the transverse area from within which a pair of photons can emerge when delocalization is engaged:

$$
\Gamma_{C}^{\prime}=S\left|e_{i} \bar{e}_{k}^{\prime} \chi_{i(j k)}^{(2)}\left(\bar{e}_{j}^{\prime}+\frac{\varepsilon_{0}}{n^{2} N^{\prime}} \bar{e}_{m}^{\prime} \chi_{l m}^{(1)} \frac{\pi C^{2} \sigma_{j l}}{N_{C}}\right)\right|^{2} .
$$

Note that in the case of very small $C$ such that there are no centers B included, $N_{\mathrm{C}}=0$ and therefore $\sigma=0$. The second term thus includes an indeterminate $0 / 0$ factor. Choosing this to be zero collapses the rate equation into the single-center rate:

$$
\Gamma_{0}^{\prime}=S\left|M_{\mathrm{A}}\right|^{2}=S\left|e_{i}{\overline{e^{\prime}}}_{k} \chi_{i(j k)}^{(2)} \bar{e}_{j}^{\prime}\right|^{2} .
$$

Also note that expanding $C$ indefinitely turns Eq. (24) into a global sum of all possible centers $B$. The rate result in this 


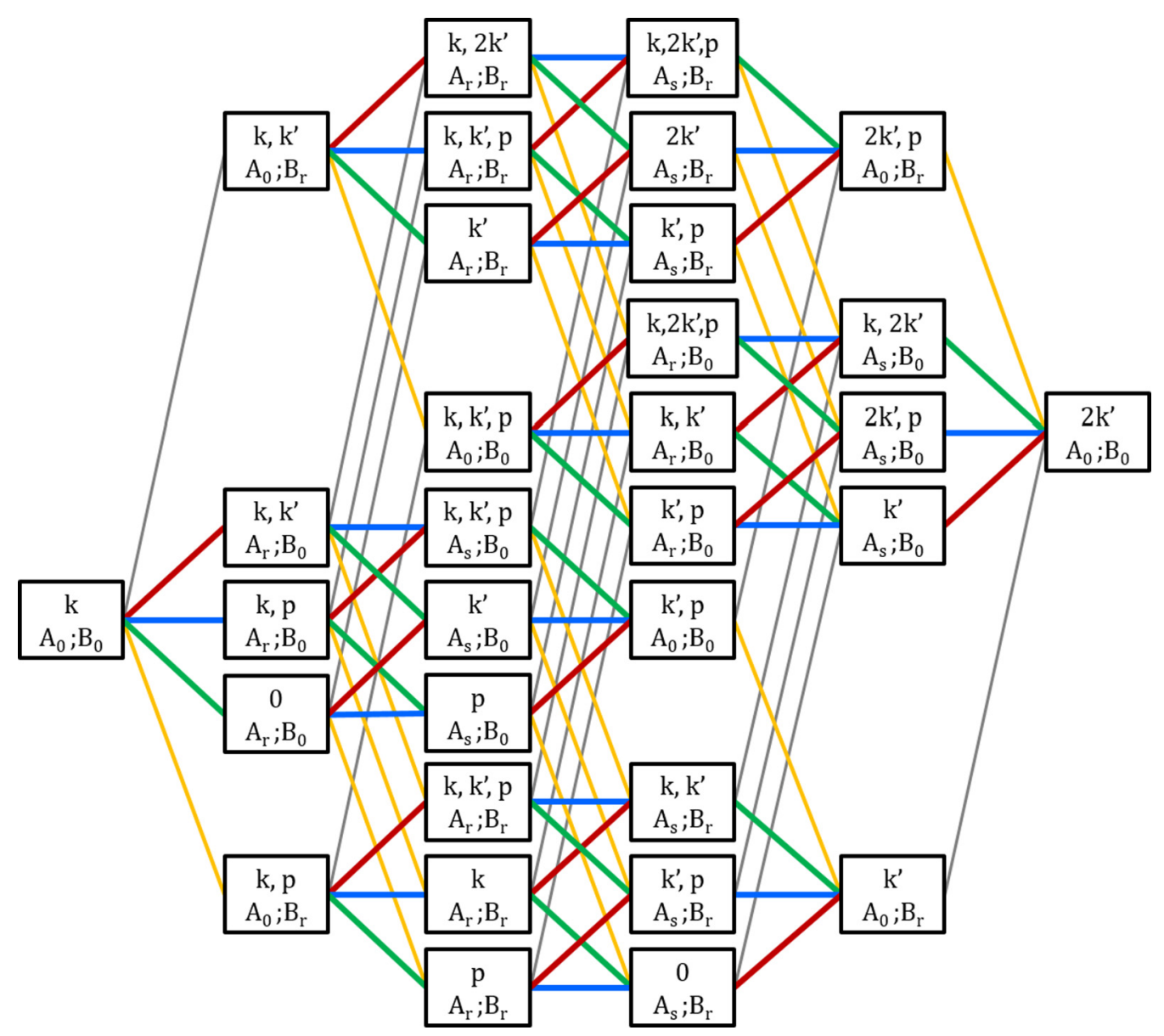

FIG. 5. State-sequence diagram for the nonlocal process. The state for the system (comprising three parts: A, B, and radiation) evolves from left to right, from state $i$ shown at the far left, to state $f$ at the far right. The process entails five distinct interaction events, each illustrated with parallel lines of a given color: absorption of the input photon by A (green), creation of an output photon $k^{\prime}$ by A (red), creation of an output photon $k^{\prime}$ by B (gray), interaction of the virtual photon $p$ by A (blue), and interaction of the virtual photon $p$ by B (gold).

case is the overall rate encompassing delocalization across all available sites. If the material is of uniform density, the ratio $\pi C^{2} \sigma_{j l} / N_{C}$ converges at the limit of infinite $C$, and therefore the overall rate $\Gamma_{\infty}^{\prime}$ can be found by the convergence of Eq. (26) at long range.

\section{RATE COMPUTATION}

In this section we describe numerical calculations of the total rate of pair production, given by the complete index summation of Eq. (26). The numerical procedure, a lattice sum calculation, follows earlier approaches for calculating quantum amplitudes associated with molecular aggregates [84,85]. Here, we expand upon a preliminary, less computationally demanding treatment of SPDC, in which results were restricted to a single element of the coupling tensor [41].

Calculations of this kind require information about the physical structure of the medium, as each term of Eq. (24) is an explicit function of B's position $\boldsymbol{r}_{\mathrm{B}}$. For computational efficiency it is convenient to approximate the medium as a primitive cubic lattice. Point $\mathrm{A}$ is an appropriate origin for the coordinate system, and an optical center B is placed at every site that has integer values for the $x, y$, and $z$ coordinates in units of the unit-cell length $u$, such that $N^{\prime}=u^{-3}$. The latter length is chosen to be approximately one-tenth of the wavelength for the output mode $\boldsymbol{k}^{\prime}$, as given by $\lambda^{\prime}=2 \pi / k^{\prime}$. The computational implementation of this calculation begins by constructing a virtual cubic lattice of $101^{3}$ positions.

Implementation of the numerical rate calculation was achieved using FORTRAN 90 with Intel compilers. This work was broken down into two phases. The first (and most computationally demanding) phase was an implementation of Eq. (24), calculating values of $\sigma_{j l}$ for all values of $C$ up to $50 u$, in increments of $0.1 u$. The results from this are illustrated in Fig. 6. It is important to note that the $\sigma_{j l}$ values feed into the quantum amplitude in the form of a tensorial product, and that it is the modulus square of the overall amplitude that determines the rate - as given by Eq. (26).

While Eq. (20) allows for the linear susceptibility to have an arbitrary second-rank tensor form, the assumption of medium isotropy allows it to be cast as a scalar such that $\chi_{l m}^{(1)}=\delta_{l m} \chi^{(1)}$. The relationship $\chi^{(1)}=|n|^{2}-1$ serves as a useful estimate for this scalar value. To secure values that give an indicative magnitude of the effects that might arise with a nonlinear optical medium typically used for SPDC, we adopt scalar nonlinear-susceptibility values for $\beta$-barium borate (BBO). It is to be understood that, as regards its linear optical susceptibility, the implications are no more than a neglect of 


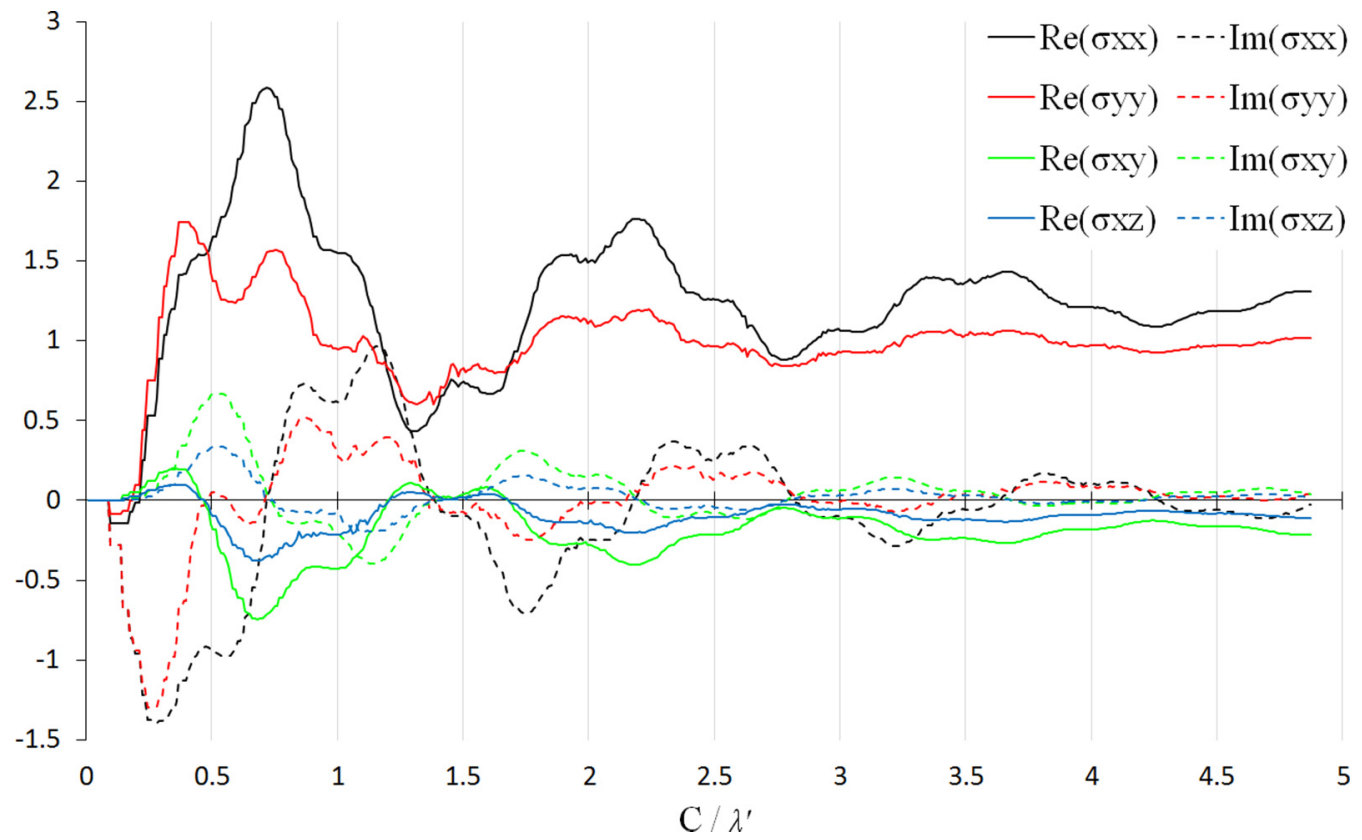

FIG. 6. The four independent components of the tensor $\sigma_{j l}(C)$, computed according to Eq. (24) in our virtual cubic lattice of $101^{3}$ positions. In order to avoid alignments that might influence the sum over positions, the direction of the radiation mode is chosen to be misaligned with the Cartesian unit vectors-in a standard basis, $\boldsymbol{k}^{\prime}=0.25 u^{-1} \hat{\mathbf{i}}+0.5 u^{-1} \hat{\mathbf{j}}+0.25 u^{-1} \hat{\mathbf{k}}$. The index symmetry of $V_{i j}$ [Eq. (21)] leads to $\sigma_{j l}=\sigma_{l j}$, and the equality $k_{x}=k_{z}$ in our choice of $\boldsymbol{k}^{\prime}$ leads to $\sigma_{x x}=\sigma_{z z}$ and $\sigma_{x y}=\sigma_{y z}$.

simple orientational features in the propensity for nonlocal down-conversion. However, for the nonlinear-susceptibility tensor $\chi_{i(j k)}^{(2)}$ that plays a central role in down-conversion, we need not entertain such approximations. If the plane of each $\mathrm{BBO}$ unit is aligned to the lattice $x y$ plane, then only four components of $\chi^{(2)}$ are significant: $\chi_{x(x x)}^{(2)}=\chi_{y(y y)}^{(2)} ; \chi_{z(x x)}^{(2)}=\chi_{z(y y)}^{(2)}$, and the other 14 components are negligible. It is expedient to use dimensionless values for these components, as the units of $\chi^{(2)}$ are absorbed by the proportionality constant $S$ in Eq. (23).

The second phase of computation utilized the $\sigma_{j l}$ data and a calculation of $N_{C}$ in an explicit implementation of Eq. (26), to calculate the rate of SPDC as a function of the cutoff radius $C$ from zero to $50 u$. The results are shown in Fig. 7 .

The nearest six centers B are at a distance of $1.0 u$ from the origin A, so the first nine data points where $C<1.0 u$ describe the limit $\Gamma_{C}^{\prime}=\Gamma_{0}^{\prime}$ according to Eq. (27). The opposite limit, $\Gamma_{\infty}^{\prime}$, is found at the convergence of $\Gamma_{C}^{\prime}$ in the region $C>4 \lambda^{\prime}$, where delocalization over more than 300000 centers B is accounted for. It is worth observing that the physically sensible convergence to a stable plateau is crucially dependent on correctly applying the normalization factor for the milticenter form of the sum over states, as in Eq. (12); otherwise, asymptotic convergence cannot be secured. Comparing the overall delocalized result to the single-center rate gives us the ratio $\Gamma^{\prime}{ }_{\infty} / \Gamma^{\prime}{ }_{0}=1.022$, meaning that the inclusion of long-range delocalization enhances the observable rate of SPDC by $2.2 \%$.

\section{DISCUSSION}

The main result of this work is concerned with the nonlocal mechanism of generating photon pairs in SPDC, and possible manifestations of these effects are discussed below. First, however, we note that few previous works have attempted to tackle, at quantum electrodynamical level, the multicenter sums required for this kind of analysis. By far the largest body of such calculations is limited to two-center interactions, as featured extensively in works by Power [86], Craig and Thirunamachandran [44], Andrews and Bradshaw [42], and Salam [45] amongst others. These are generally calculations that concern the energies, forces, and spectroscopic transitions associated with specifically pairwise interactions. To take one recent example [87], an article on medium-modified resonance energy transfer (RET) cast the effects in terms of pairwise coupling between the nearest individual molecules of the bulk; i.e., $N=2$. For all such cases the $(N-1)^{-1}$ factor that arises in the appropriate counterpart to Eq. (12) is simply unity, and no notice needs to be taken of it. There are a limited number of reports on explicit three-molecule calculations using QED [88,89], for which lattice sums were irrelevant. Following some earlier work in terms of dopant concentrations [90], an explicit summing of quantum amplitudes over crystallographic lattices of various kinds appears in the work of Leeder and Andrews [91], which did not engage the full analysis reported here.

The results that we have secured confirm and consolidate our preliminary results identifying a potentially significant mechanism for the nonlocal production of down-converted photon pairs, [41] now fully accounting for the multicenter sums. The unique aspect of this work extends from an advancement of the underlying theory, through a more substantial computational analysis, to results that indicate an effect that is meaningfully within the reach of experimental detection-the possibility of one photon pair in every 50 or so emerging from close but physically distinct locations. Of course, for different materials, different axial orientations, and different optical frequencies, the results could be quite different-but 


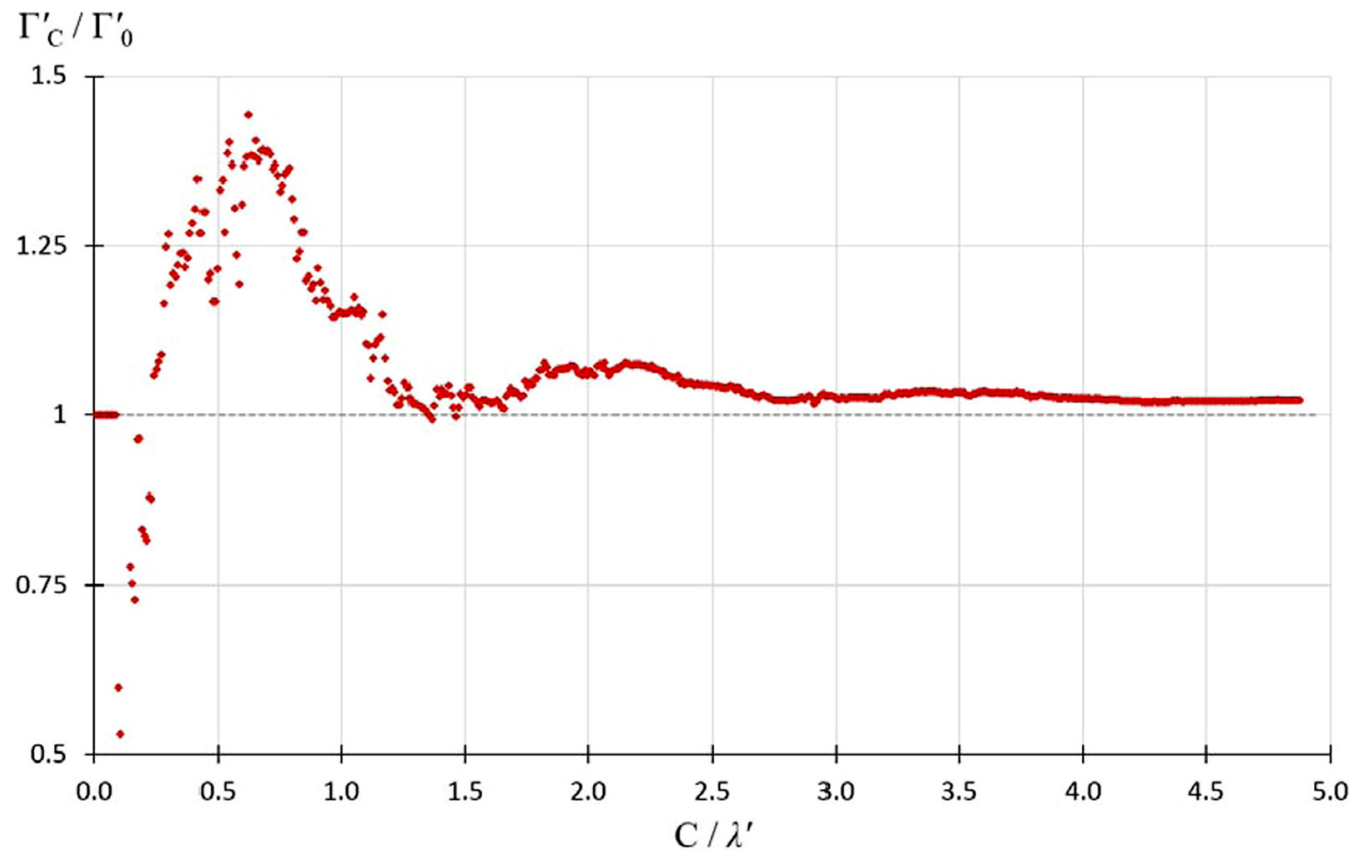

FIG. 7. The observable rate of SPDC, $\Gamma_{C}^{\prime}$, computed according to Eq. (26) using the $\sigma$ results shown in Fig. 6, and normalized against the $C=0$ rate given by Eq. (27). Values for the material parameters are chosen to approximately match BBO: $n \cong 1.7+0.1 i ; \chi_{x(x x)}^{(2)}=5.8$ and $\chi_{z(x x)}^{(2)}=0.29$ (manufacturer reported values relative to standard KDP reference) [101].

the magnitudes of the computational parameters in our quantitative analysis are realistically representative: Some systems could exhibit less prominent nonlocal effects, but others could indeed give such effects even greater prominence.

In terms of physical significance, the dampened oscillatory form of behavior exhibited in Fig. 7 merits a number of comments. Although the results should be taken within the context of a rectangular lattice model, several interesting insights arise, and may lead to more sophisticated numerical models. It is worth noting that the abscissa scale meaningfully signifies not only the effects of expanding the scale of the calculation, to distances more and more remote from the site of input photon annihilation: it can also be understood to represent the actual effect of increasing the size of nanoparticles in their capacity to produce down-conversion, including provision for the nonlocal mechanism. In this respect the rise in efficiency that runs out to about half an output wavelength means that the efficiency of down-conversion actually increases with particle size, up to a limit that represents an optimum size, where the propensity for SPDC is markedly larger per unit volume than a bulk of the same material. Hence, essentially due to local effects of constructive optical interference, it is possible for compacted nanoparticles of similar dimensions to exhibit enhanced nonlinear optical activity, compared to the bulk. An analogous manifestation of coherence came to light in connection with second-harmonic generation (the time reversal of SPDC) in nanomaterials [92-95]. Beyond this point, the trailing off to an asymptotic limit signifies that as the sample size grows beyond a couple of wavelengths, the scale of such advantages is quickly lost-because additional contributions presenting destructive interference come into play. Eventually, any individual particle becomes more and more representative of the bulk phase. The nonlinear conver- sion efficiency plateaus-but still the efficiency is higher than would be expected from entirely localized pair emission.

Several features of nonlocal origin can be expected to be detectable in the output. First, one can anticipate a degree of temporal broadening, associated with some photon pairs being emitted from positions that are within a wavelength or so in advance of, or behind, each other with respect to the forward emission direction. The corresponding lateral broadening is also interesting; it signifies that in applications such as "ghost imaging" [33,96-98], the resolution of the image will be to some extent compromised by an insurmountable phenomenon of quantum origin — notwithstanding experimental limitations associated with physical optics [99] — because perfect imaging would require each photon pair to originate from precisely the same spatial origin. This effect, if the nonlocal mechanism had not been identified, might indeed be interpreted as simply manifesting a position-momentum quantum uncertainty with respect to directions perpendicular to the down-converted photon propagation. It is also interesting to conjecture whether effects of a similar kind might play a role in the creation of two-photon states through interactions between Rydberg atoms [100]. It remains to be seen what other forms of photon-pair nonlocality might prove amenable to experimental determination; the possible implications for quantum entanglement between emitted photons is a subject now under further active investigation.

\section{ACKNOWLEDGMENTS}

We are pleased to acknowledge helpful comments from our colleague Dr. David S. Bradshaw. The calculations presented in this paper were carried out on the High Performance Computing Cluster supported by the Research and Specialist Computing Support service at the University of East Anglia. 
[1] P. G. Kwiat, K. Mattle, H. Weinfurter, A. Zeilinger, A. V. Sergienko, and Y. Shih, New High-Intensity Source of Polarization-Entangled Photon Pairs, Phys. Rev. Lett. 75, 4337 (1995).

[2] S. P. Walborn, C. Monken, S. Pádua, and P. S. Ribeiro, Spatial correlations in parametric down-conversion, Phys. Rep. 495, 87 (2010).

[3] M. Müller, S. Bounouar, K. D. Jöns, M. Glässl, and P. Michler, On-demand generation of indistinguishable polarization-entangled photon pairs, Nat. Photonics 8, 224 (2014).

[4] R. Horodecki, P. Horodecki, M. Horodecki, and K. Horodecki, Quantum entanglement, Rev. Mod. Phys. 81, 865 (2009).

[5] J. T. Barreiro, N. K. Langford, N. A. Peters, and P. G. Kwiat, Generation of Hyperentangled Photon Pairs, Phys. Rev. Lett. 95, 260501 (2005).

[6] M.-X. Dong, W. Zhang, S. Shi, K. Wang, Z.-Y.Zhou, S.-L. Liu, D.-S. Ding, and B.-S. Shi, Two-color hyper-entangled photon pairs generation in a cold $85 \mathrm{Rb}$ atomic ensemble, Opt. Express 25, 10145 (2017).

[7] D. L. Andrews and P. Allcock, Optical Harmonics in Molecular Systems (Wiley-VCH, Weinheim, 2002).

[8] R. W. Boyd, Nonlinear Optics (Academic Press, New York, 2003).

[9] K. Edamatsu, Entangled photons: Generation, observation, and characterization, Jpn. J. Appl. Phys. 46, 7175 (2007).

[10] D. S. Simon, G. Jaeger, and A. V. Sergienko, Quantum Metrology, Imaging, and Communication, Quantum Science and Technology (Springer, Berlin, 2017).

[11] C. H. Bennett, Quantum information, Phys. Scr. T76, 210 (1998).

[12] M. A. Nielsen and I. Chuang, Quantum Computation and Quantum Information (Cambridge University Press, Cambridge, 2002).

[13] M. M. Wilde, Quantum Information Theory (Cambridge University Press, Cambridge, 2013).

[14] N. Gisin, G. Ribordy, W. Tittel, and H. Zbinden, Quantum cryptography, Rev. Mod. Phys. 74, 145 (2002).

[15] C. H. Bennett and G. Brassard, Quantum cryptography: Public key distribution and coin tossing, Theor. Comput. Sci. 560, 7 (2014).

[16] C. H. Bennett, G. Brassard, C. Crépeau, R. Jozsa, A. Peres, and W. K. Wootters, Teleporting an Unknown Quantum State via Dual Classical and Einstein-Podolsky-Rosen Channels, Phys. Rev. Lett. 70, 1895 (1993).

[17] D. Bouwmeester, J.-W. Pan, K. Mattle, M. Eibl, H. Weinfurter, and A. Zeilinger, Experimental quantum teleportation, Nature 390, 575 (1997).

[18] M. Barrett, J. Chiaverini, T. Schaetz, J. Britton, W. Itano, J. Jost, E. Knill, C. Langer, D. Leibfried, and R. Ozeri, Deterministic quantum teleportation of atomic qubits, Nature 429, 737 (2004).

[19] A. Steane, Quantum computing, Rep. Prog. Phys. 61, 117 (1998).

[20] S. Barz, J. F. Fitzsimons, E. Kashefi, and P. Walther, Experimental verification of quantum computation, Nat. Phys. 9, 727 (2013).

[21] M. Hirvensalo, Quantum Computing (Springer, Berlin, 2013).
[22] M. Blasone, F. Dell'Anno, S. De Siena, and F. Illuminati, Entanglement in neutrino oscillations, EPL 85, 50002 (2009).

[23] J. Formaggio, D. Kaiser, M. Murskyj, and T. Weiss, Violation of the Leggett-Garg Inequality in Neutrino Oscillations, Phys. Rev. Lett. 117, 050402 (2016).

[24] B. Hensen, H. Bernien, A. E. Dréau, A. Reiserer, N. Kalb, M. S. Blok, J. Ruitenberg, R. F. Vermeulen, R. N. Schouten, and C. Abellán, Loophole-free Bell inequality violation using electron spins separated by 1.3 kilometres, Nature 526, 682 (2015).

[25] V. Vedral, Quantifying entanglement in macroscopic systems, Nature 453, 1004 (2008).

[26] X.-L. Wang, L.-K. Chen, W. Li, H.-L. Huang, C. Liu, C. Chen, Y.-H. Luo, Z.-E. Su, D. Wu, and Z.-D. Li, Experimental Ten-Photon Entanglement, Phys. Rev. Lett. 117, 210502 (2016).

[27] A. Aspect, P. Grangier, and G. Roger, Experimental Tests of Realistic Local Theories via Bell's theorem, Phys. Rev. Lett. 47, 460 (1981).

[28] A. Aspect, J. Dalibard, and G. Roger, Experimental Test of Bell's Inequalities Using Time-Varying Analyzers, Phys. Rev. Lett. 49, 1804 (1982).

[29] A. Aspect, P. Grangier, and G. Roger, Experimental Realization of Einstein-Podolsky-Rosen-Bohm Gedankenexperiment: A New Violation of Bell's Inequalities, Phys. Rev. Lett. 49, 91 (1982).

[30] A. S. Solntsev, A. A. Sukhorukov, D. N. Neshev, and Y. S. Kivshar, Spontaneous Parametric Down-Conversion and Quantum Walks in Arrays of Quadratic Nonlinear Waveguides, Phys. Rev. Lett. 108, 023601 (2012).

[31] H. Jin, F. M. Liu, P. Xu, J. L. Xia, M. L. Zhong, Y. Yuan, J. W. Zhou, Y. X. Gong, W. Wang, and S. N. Zhu, On-Chip Generation and Manipulation of Entangled Photons Based on Reconfigurable Lithium-Niobate Waveguide Circuits, Phys. Rev. Lett. 113, 103601 (2014).

[32] M. McLaren, T. Mhlanga, M. J. Padgett, F. S. Roux, and A. Forbes, Self-healing of quantum entanglement after an obstruction, Nat. Commun. 5, 3248 (2014).

[33] T. Pittman, Y. Shih, D. Strekalov, and A. Sergienko, Optical imaging by means of two-photon quantum entanglement, Phys. Rev. A 52, R3429(R) (1995).

[34] J. L. O'Brien, G. J. Pryde, A. G. White, T. C. Ralph, and D. Branning, Demonstration of an all-optical quantum controlledNOT gate, Nature 426, 264 (2003).

[35] M. P. Edgar, D. S. Tasca, F. Izdebski, R. E. Warburton, J. Leach, M. Agnew, G. S. Buller, R. W. Boyd, and M. J. Padgett, Imaging high-dimensional spatial entanglement with a camera, Nat. Commun. 3, 984 (2012).

[36] M. Giustina, M. A. Versteegh, S. Wengerowsky, J. Handsteiner, A. Hochrainer, K. Phelan, F. Steinlechner, J. Kofler, J.-Å. Larsson, and C. Abellán, Significant-Loophole-Free Test of Bell's Theorem with Entangled Photons, Phys. Rev. Lett. 115, 250401 (2015).

[37] L. K. Shalm, E. Meyer-Scott, B. G. Christensen, P. Bierhorst, M. A. Wayne, M. J. Stevens, T. Gerrits, S. Glancy, D. R. Hamel, and M. S. Allman, Strong Loophole-Free Test of Local Realism, Phys. Rev. Lett. 115, 250402 (2015).

[38] D. Walls, Quantum theory of nonlinear optical phenomena, J. Phys. A: Gen. Phys. 4, 813 (1971). 
[39] G. Grynberg, A. Aspect, and C. Fabre, Introduction to Quantum Optics: From the Semi-Classical Approach to Quantized Light (Cambridge University Press, Cambridge, 2010).

[40] P. D. Drummond and M. Hillery, The Quantum Theory of Nonlinear Optics (Cambridge University Press, Cambridge, 2014).

[41] K. A. Forbes, J. S. Ford, and D. L. Andrews, Nonlocalized Generation of Correlated Photon Pairs in Degenerate DownConversion, Phys. Rev. Lett. 118, 133602 (2017).

[42] D. L. Andrews and D. S. Bradshaw, The role of virtual photons in nanoscale photonics, Ann. Phys. (Berlin, Ger.) 526, 173 (2014).

[43] W. P. Healy, Non-relativistic Quantum Electrodynamics (Academic Press Inc., London, 1982).

[44] D. P. Craig and T. Thirunamachandran, Molecular Quantum Electrodynamics: An Introduction to Radiation-Molecule Interactions (Dover Publications, Mineola, NY, 1998).

[45] A. Salam, Molecular Quantum Electrodynamics. Long-Range Intermolecular Interactions (Wiley, Hoboken, NJ, 2010).

[46] M. Hillery, An Introduction to the quantum theory of nonlinear optics, Acta Phys. Slovaca 59, 1 (2009).

[47] H. B. G. Casimir and D. Polder, The influence of retardation on the London-van der Waals forces, Phys. Rev. 73, 360 (1948).

[48] O. M. Maragò, P. H. Jones, P. G. Gucciardi, G. Volpe, and A. C. Ferrari, Optical trapping and manipulation of nanostructures, Nat. Nanotechnol. 8, 807 (2013).

[49] D. S. Bradshaw and D. L. Andrews, Chiral discrimination in optical trapping and manipulation, New J. Phys. 16, 103021 (2014).

[50] D. S. Bradshaw, K. A. Forbes, J. M. Leeder, and D. L. Andrews, Chirality in optical trapping and optical binding, Photonics $\mathbf{2}$, 483 (2015).

[51] D. L. Andrews and D. Bradshaw, Optical Nanomanipulation (Morgan \& Claypool Publishers, San Rafael, CA, 2016).

[52] S. E. S. Spesyvtseva and K. Dholakia, Trapping in a material world, ACS Photonics 3, 719 (2016).

[53] T. Thirunamachandran, Intermolecular interactions in the presence of an intense radiation field, Mol. Phys. 40, 393 (1980).

[54] A. Salam, Intermolecular interactions in a radiation field via the method of induced moments, Phys. Rev. A 73, 013406 (2006).

[55] L. C. Dávila Romero, J. Rodríguez, and D. L. Andrews, Electrodynamic mechanism and array stability in optical binding, Opt. Commun. 281, 865 (2008).

[56] K. Dholakia and P. Zemánek, Colloquium: Gripped by light: Optical binding, Rev. Mod. Phys. 82, 1767 (2010).

[57] V. Demergis and E.-L. Florin, Ultrastrong optical binding of metallic nanoparticles, Nano Lett. 12, 5756 (2012).

[58] Z. Yan, S. K. Gray, and N. F. Scherer, Potential energy surfaces and reaction pathways for light-mediated self-organization of metal nanoparticle clusters, Nat. Commun. 5, 3751 (2014).

[59] P. Coullet, L. Gil, and F. Rocca, Optical vortices, Opt. Commun. 73, 403 (1989).

[60] L. Allen, M. W. Beijersbergen, R. J. C. Spreeuw, and J. P. Woerdman, Orbital angular momentum of light and the transformation of Laguerre-Gaussian laser modes, Phys. Rev. A 45, 8185 (1992).

[61] M. D. Williams, M. M. Coles, K. Saadi, D. S. Bradshaw, and D. L. Andrews, Optical Vortex Generation from Molec- ular Chromophore Arrays, Phys. Rev. Lett. 111, 153603 (2013).

[62] M. D. Williams, M. M. Coles, D. S. Bradshaw, and D. L. Andrews, Direct generation of optical vortices, Phys. Rev. A 89, 033837 (2014).

[63] E. A. Power and S. Zienau, Coulomb gauge in non-relativistic quantum electro-dynamics and the shape of spectral lines, Philos. Trans. R. Soc., A 251, 427 (1959).

[64] R. Woolley, Molecular quantum electrodynamics, Proc. R. Soc. A 321, 557 (1971).

[65] E. A. Power and T. Thirunamachandran, The multipolar Hamiltonian in radiation theory, Proc. R. Soc. A 372, 265 (1980).

[66] E. A. Power and T. Thirunamachandran, On the nature of Hamiltonian for interaction of radiation with atoms and molecules: $(e / m c) p \cdot \mathrm{A},-\mu \cdot \mathrm{E}$, and all that, Am. J. Phys. 46, 370 (1978).

[67] E. A. Power and T. Thirunamachandran, Quantum electrodynamics with non-relativistic sources 1 . Transformation to the multipolar formalism for second-quantized electron and Maxwell interacting fields, Phys. Rev. A 28, 2649 (1983).

[68] E. Power and T. Thirunamachandran, Time dependence of operators in minimal and multipolar nonrelativistic quantum electrodynamics. II. Analysis of the functional forms of operators in the two frameworks, Phys. Rev. A 60, 4936 (1999).

[69] E. Power and T. Thirunamachandran, Time dependence of operators in minimal and multipolar nonrelativistic quantum electrodynamics. I. Electromagnetic fields in the neighborhood of an atom, Phys. Rev. A 60, 4927 (1999).

[70] K. A. Forbes and D. L. Andrews, Chiral discrimination in optical binding, Phys. Rev. A 91, 053824 (2015).

[71] M. D. Williams, J. S. Ford, and D. L. Andrews, Hyper-Rayleigh scattering in centrosymmetric systems, J. Chem. Phys. 143, 124301 (2015).

[72] K. A. Forbes, D. S. Bradshaw, and D. L. Andrews, Identifying diamagnetic interactions in scattering and nonlinear optics, Phys. Rev. A 94, 033837 (2016).

[73] G. Juzeliūnas and D. L. Andrews, Quantum electrodynamics of resonant energy-transfer in condensed matter, Phys. Rev. B 49, 8751 (1994).

[74] G. Juzeliūnas and D. L. Andrews, Quantum electrodynamics of resonant energy-transfer in condensed matter. II. Dynamical aspects, Phys. Rev. B 50, 13371 (1994).

[75] G. Juzeliūnas, Microscopic theory of quantization of radiation in molecular dielectrics: Normal-mode representation of operators for local and averaged (macroscopic) fields, Phys. Rev. A 53, 3543 (1996).

[76] G. Juzeliūnas, Microscopic theory of quantization of radiation in molecular dielectrics. 2. Analysis of microscopic field operators, Phys. Rev. A 55, 929 (1997).

[77] P. Löwdin and C. Wilcox, Perturbation Theory and its Application in Quantum Mechanics (Wiley, New York, 1966), p. 255.

[78] R. P. Feynman, Space-time approach to quantum electrodynamics, Phys. Rev. 76, 769 (1949).

[79] J. Ward, Calculation of nonlinear optical susceptibilities using diagrammatic perturbation theory, Rev. Mod. Phys. 37, 1 (1965).

[80] R. Wallace, Diagrammatic perturbation theory of multiphoton transitions, Mol. Phys. 11, 457 (1966). 
[81] R. D. Jenkins, D. L. Andrews, and L. C. Dávila Romero, A new diagrammatic methodology for non-relativistic quantum electrodynamics, J. Phys. B: At., Mol. Opt. Phys. 35, 445 (2002).

[82] D. L. Andrews and D. S. Bradshaw, Virtual photons, dipole fields and energy transfer: A quantum electrodynamical approach, Eur. J. Phys. 25, 845 (2004).

[83] G. Juzeliūnas and D. L. Andrews, Quantum electrodynamics of resonance energy transfer, Adv. Chem. Phys. 112, 357 (2000).

[84] J. E. Frost and G. A. Jones, A quantum dynamical comparison of the electronic couplings derived from quantum electrodynamics and Förster theory: Application to 2D molecular aggregates, New J. Phys. 16, 113067 (2014).

[85] M. P. E. Lock, D. L. Andrews, and G. A. Jones, On the nature of long range electronic coupling in a medium: Distance and orientational dependence for chromophores in molecular aggregates, J. Chem. Phys. 140, 044103 (2014).

[86] E. A. Power and T. Thirunamachandran, Casimir-Polder potential as an interaction between induced dipoles, Phys. Rev. A 48, 4761 (1993).

[87] D. L. Andrews and J. S. Ford, Resonance energy transfer: Influence of neighboring matter absorbing in the wavelength region of the acceptor, J. Chem. Phys. 139, 014107 (2013).

[88] R. Passante, E. A. Power, and T. Thirunamachandran, Radiation-molecule coupling using dynamic polarizabilities: Application to many-body forces, Phys. Lett. A 249, 77 (1998).

[89] A. Salam, Dispersion potential between three-bodies with arbitrary electric multipole polarizabilities: Molecular QED theory, J. Chem. Phys. 140, 044111 (2014).

[90] D. L. Andrews and R. D. Jenkins, A quantum electrodynamical theory of three-center energy transfer for upconversion and downconversion in rare earth doped materials, J. Chem. Phys. 114, 1089 (2001).
[91] J. M. Leeder and D. L. Andrews, Enhancing optical upconversion through electrodynamic coupling with ancillary chromophores, J. Phys. Chem. C 118, 23535 (2014).

[92] D. L. Andrews, P. Allcock, and A. A. Demidov, Theory of second-harmonic generation in randomly oriented species, Chem. Phys. 190, 1 (1995).

[93] P. Allcock, D. L. Andrews, S. R. Meech, and A. J. Wigman, Doubly forbidden second-harmonic generation from isotropic suspensions: Studies on the purple membrane of Halobacteriumhalobium, Phys. Rev. A 53, 2788 (1996).

[94] D. L. Andrews and L. C. Dávila Romero, Local coherence and the temporal development of second harmonic emission, J. Phys. B: At., Mol. Opt. Phys. 34, 2177 (2001).

[95] W.-K. Zhang, H.-F. Wang, and D.-S. Zheng, Quantitative measurement and interpretation of optical second harmonic generation from molecular interfaces, Phys. Chem. Chem. Phys. 8, 4041 (2006).

[96] A. Gatti, E. Brambilla, M. Bache, and L. A. Lugiato, Ghost Imaging with Thermal Light: Comparing Entanglement and Classical Correlation, Phys. Rev. Lett. 93, 093602 (2004).

[97] P. A. Morris, R. S. Aspden, J. E. Bell, R. W. Boyd, and M. J. Padgett, Imaging with a small number of photons, Nat. Commun. 6, 5913 (2015).

[98] J. Cheng, Theory of ghost scattering with biphoton states, Photonics Res. 5, 41 (2017).

[99] M. H. Rubin and Y. Shih, Resolution of ghost imaging for nondegenerate spontaneous parametric down-conversion, Phys. Rev. A 78, 033836 (2008).

[100] J. Ruseckas, I. Yu, and G. Juzeliūnas, Creation of two-photon states via interactions between Rydberg atoms during light storage, Phys. Rev. A 95, 023807 (2017).

[101] http://www.redoptronics.com/BBO-crystal.html. 